\title{
Translittérations féeriques au Moyen Age :de Mélior à Mélusine, entre histoire et fiction
}

Jean-Claude Mühlethaler

\section{(2) OpenEdition}

\section{Journals}

Édition électronique

URL : http://journals.openedition.org/edl/198

DOI : 10.4000/edl. 198

ISSN : 2296-5084

Éditeur

Université de Lausanne

\section{Édition imprimée}

Date de publication : 15 décembre 2011

Pagination : 167-190

ISBN : 978-2-940331-26-0

ISSN : 0014-2026

\section{Référence électronique}

Jean-Claude Mühlethaler, «Translittérations féeriques au Moyen Age :de Mélior à Mélusine, entre histoire et fiction », Études de lettres [En ligne], 3-4 | 2011, mis en ligne le 15 décembre 2014, consulté le 19 décembre 2020. URL : http://journals.openedition.org/edl/198 ; DOI : https://doi.org/10.4000/ edl.198 


\section{TRANSLITTÉRATIONS FÉERIQUES AU MOYEN AGE: DE MÉLIOR À MÉLUSINE, ENTRE HISTOIRE ET FICTION}

Déjà le récit de Cupidon et Psyché dans l'Asinus aureus pose le problème du genre de cette histoire enchâssée, racontée par une vieille - est-ce un mythe ou une fable philosophique? - et, par voie de conséquence, celui du statut même des dieux qui y interviennent. Qu'on voie dans Partenopeu de Blois un lointain avatar du récit d'Apulée, dans lequel la fée se substitue à Cupidon, ou qu'on le rattache au folklore (celtique), la question est sensiblement la même au XII ${ }^{\mathrm{e}}$ siècle: les auteurs médiévaux croyaient-ils aux «êtres faés» ou ceux-ci étaient-ils à leurs yeux les acteurs imposés par le choix d'un certain univers fictionnel? Si la critique est fondamentalement d'accord pour parler d'une «rationalisation» ou d'une "moralisation" de la fée, la démarche par laquelle l'écrivain médiéval adapte et actualise le matériau issu d'une tradition est susceptible de subtiles variations: la «réécriture» est chaque fois tributaire du projet d'écriture respectif, qu'il s'agisse de Partenopeu de Blois ou du lai de Guigemar de Marie de France. Au-delà du statut de la fée et de l'altérité qu'elle représente, c'est le statut même de la femme et du héros qui sont en cause, leur place et leur fonction au sein de la société féodale. La «translittération» de parcours narratifs stéréotypés, voire universaux, n’a ici rien à voir avec la récupération politique du folklore, transmis par voie orale, à laquelle s'attelle, quelque deux siècles plus tard, Jean d'Arras dans sa Mélusine: il extrait la légende de la fée poitevine du domaine de la fabula en inscrivant la merveille dans l'histoire, la région et l'expérience vécue pour légitimer les droits du duc de Berry sur Lusignan.

Monde à l'envers, monde exemplaire, la féerie est critique de la réalité durcie. Elle ne demeure pas à côté de celle-ci, elle réagit sur elle; elle invite à la transformer, à remettre à l'endroit ce qui, en elle, est mal placé.

Michel Butor, «La Balance des fées», p. 65. 
Le roman de Partenopeu de Blois est l'œuvre d'un contemporain ${ }^{1}$ anonyme de Chrétien de Troyes. Même si un lecteur moderne est peu sensible aux motifs qui, selon les spécialistes, se font écho chez les deux auteurs, il n'en découvrira pas moins des éléments familiers dans le récit des amours de Mélior et de Partenopeu. C'est l'histoire d'un jeune chevalier, favori du roi de France, qu'un sanglier entraîne dans les profondeurs de la forêt des Ardennes, de sorte qu'il se retrouve seul, loin de ses compagnons. Perdu, il erre dans des bois hantés par des serpents, des vouivres et des dragons ailés, puis arrive sur les rives de la mer où l'attend une nef sans équipage qui le transporte jusqu'à Chief d'Oirre. La ville est d'une richesse merveilleuse et un plantureux repas sera servi au jeune homme dans un palais par des personnes invisibles. La nuit, alors qu'il est couché, une "arme» ${ }^{2}$ - c'est-à-dire un être indéfini, un esprit - vient le rejoindre dans son lit. Bien que Partenopeu ne voie rien dans l'obscurité, il remarquera qu'il s'agit d'une jeune femme, s'enhardira peu à peu et passera à l'acte. L'inconnue lui révèle alors son nom - "Melior» (v. 1763) -, lui promet de grandes richesses, mais lui interdit de chercher à la voir. Evidemment, l'interdit sera transgressé, suivant un parcours narratif récurrent dans les récits médiévaux qui sont souvent marqués par un substrat folklorique diffus ${ }^{3}$ que les auteurs adaptent et inscrivent dans les discours en vogue dans leur communauté de référence ${ }^{4}$. Sur l'instigation de sa mère, Partenopeu contemple son amie une nuit à «la clarté / De la lanterne» (v. 4512-4513). Mélior se réveille et lui révèle qu’à cause de son geste fatal, elle ne peut plus protéger leur amour du regard des autres. Le matin, les deux amants sont découverts, le scandale éclate au grand jour et Partenopeu est chassé de Chief d'Oirre.

Un lecteur moderne reconnaîtra dans le parcours narratif de Partenopeu de Blois une "translittération ${ }^{5}$ de l'histoire de Cupidon et

I. Sur la date controversée du roman, voir A. Reynders, «Le Roman de Partenopeu de Blois est-il l'œuvre d'un précurseur de Chrétien de Troyes?».

2. Partenopeu de Blois, v. 1121.

3. Voir à ce sujet F. Wolfzettel, Le Conte en palimpseste, p. 16-32.

4. C'est la démarche qu'U. Heidmann et J.-M. Adam désignent par le terme de "généricité» dans Textualité et intertextualité des contes, p. 19-20.

5. Le terme de «translittération», emprunté à E. W. Harries (Twice upon a Time, p. 135-139) et celui de "réécriture», dû à D. Poirion ("Ecriture et ré-écriture au Moyen Age») se recoupent dans une large mesure. Pour notre propos, "translittération » a pour avantage de mettre l'accent sur la transposition-transformation de patterns (parcours 
de Psyché d'Apulée avec une inversion des rôles masculin et féminin: la fée occupe la place du dieu, le chevalier celle de la mortelle. Bien que l'Asinus aureus soit cité par saint Augustin ${ }^{6}$ et que Martianus Capella ait commenté les amours de Cupidon et Psyché dans le De nuptiis Philologiae et Mercurii, il est peu probable que le roman médiéval s'en soit directement inspiré. Apulée n'est guère lu au Moyen Age et les frères Grimm ont trouvé plusieurs récits à structure comparable dans le folklore $^{7}$. Différents éléments figuratifs communs établissent malgré tout une troublante proximité entre Partenopeu de Blois et le texte antique: la nef merveilleuse se substitue à la tendre brise de Zéphyr; les splendeurs orientales de Chief d'Oirre rappellent le luxe du palais d'Amour et annoncent, comme d'ailleurs le repas servi par des êtres invisibles, La Belle et la Bête de Madame de Villeneuve ou de Madame Leprince de Beaumont, voire leur variante russe, La Fleur écarlate de Sergueï Aksakov. Chez Apulée aussi bien que chez l'auteur médiéval, l'interdit imposé par l'être surnaturel frappe la vue et les scènes de transgression nocturnes se font écho; chaque fois, ce sont des parents proches - les sœurs ou la mère - qui provoquent la catastrophe.

Dans la figurativité de l'œuvre du XII ${ }^{\mathrm{e}}$ siècle, le médiéviste décèle d'abord les traces de l'héritage celtique dont s'inspirent à l'époque les récits arthuriens: la forêt avec son bestiaire fabuleux, l'animal-guide surgi de nulle part, puis le "navire errant désert ${ }^{8}$ qui entraîne le héros loin de son pays natal vers un Ailleurs paradisiaque ${ }^{9}$ où le temps s'abolit. Les indices convergent pour suggérer la présence du surnaturel et on s'attend à voir apparaître la merveille: la dame de Chief d'Oirre ne saurait être qu'une fée toute-puissante!... C'est pourtant de «damoisele» ${ }^{10}$ que le texte qualifie Mélior, quand elle se glisse dans le lit du jeune homme, comme s'il s'agissait d'une quelconque jeune femme noble. On lui enlève ainsi une part de son inquiétante étrangeté, car le terme de fée

narratifs). Mais ne serait-il pas temps de promouvoir le dialogue entre les disciplines au lieu que chacun forge ses propres instruments de travail?

6. Augustin, De civitate Dei, 18.18 .

7. Aux yeux de L. Harf-Lancner (Les Fées au Moyen Age, p. 317-328), l'influence du folklore est décisive. C'est aussi l'opinion de V. Gély, L'Invention d'un mythe, p. 17-18 et p. 284-285.

8. L'expression est d'Y. Bonnefoy, «L'Attrait des romans bretons", p. 123.

9. A Chief d'Oirre, le héros croit être «en paradis» (Partenopeu de Blois, v. 874).

Io. Ibid., v. 1129,1139 et 1159 . 
a des connotations négatives dans Partenopeu de Blois. Il apparaît seulement dans la bouche des opposants qui cherchent à détourner le héros d'un amour néfaste à leurs yeux. Ainsi, la nièce du roi de France enivre Partenopeu et tente de le séduire par le plaisir des sens. Alors qu'elle croit avoir gagné la partie, elle s'exclame:

Jetés estes de la baillie

La bele fee, vostre amie ${ }^{11}$.

Vous voilà libéré du joug

De la belle fée, votre amie.

Ce sont ces mots mêmes, et avant tout le terme d'amie, qui font prendre conscience à Partenopeu qu'il est sur le point de trahir Mélior. Saisi de remords, il s'en retourne au plus vite chez son amie et il faudra une seconde tentative pour l'amener à transgresser l'interdit. Craignant pour le salut de Partenopeu, sa mère fait venir l'évêque de Paris et lui révèle que son fils a été enlevé par une "fee» (v. 4355). De même que les sœurs de Psyché laissent entendre à la jeune fille qu'elle s'unit à un serpent monstrueux ${ }^{12}$, la mère et l'évêque s'ingénient à diaboliser l'être surnaturel: il s'agirait d'un «maufés» (v. 4462), d'un diable, dont l'obscurité cacherait la laideur innommable. Ils réveillent ainsi chez Partenopeu les terreurs du premier jour passé à Chief d'Oirre: les merveilles de la ville ne lui semblaient-elles alors pas être une œuvre de «faerie» (v. 809), le repas fastueux «songe» ou «fantosme» (v. 916)? La rime «fable»/«diable» ${ }^{13}$ dit jusqu'où va l'angoisse du jeune homme face à un spectacle qui défie les sens et la raison, lui faisant craindre d'être «engeniés» (v. 930), d'être victime d'un «engin», c'est-à-dire d'une ruse diabolique qui joue sur les apparences.

On retrouve ainsi chez Partenopeu cette «incertitude fantastique» ${ }^{14}$ de l'homme amené aux confins de l'illusion et de la réalité, ce mélange de crainte et d'attirance que connaît le sujet confronté à l'altérité

II. Ibid., v. 4049-4050.

I2. Apulée, Métamorphoses, 5.17.

13. Partenopeu de Blois, v. 983-984.

I4. L'expression est de F. Dubost, Aspects fantastiques de la littérature narrative médiévale, vol. I, p. 380. L'ambivalence de la figure de la fée trouve sa source dans les Fata antiques, et elle traverse l'histoire du conte de fées. Voir par exemple l'article de M. Viegnes sur les fées fin-de-siècle dans le présent volume. 
merveilleuse dans les récits du Moyen Age ${ }^{15}$. Dans ce contexte, la première nuit d'amour surprend le lecteur, car il s'attend à une fée séductrice et entreprenante qui, comme dans Le Lai de Lanval de Marie de France, va offrir généreusement son corps et ses richesses au héros ${ }^{16}$, comblant sur-le-champ toutes ses attentes. Mais Mélior se présente en pucelle effarouchée, avant de céder aux avances de plus en plus hardies du jeune homme dans une scène crue qui tient du viol. La fée se retrouve ici dans la situation de la bergère dont le chevalier profite dans certaines "pastourelles» ${ }^{17}$, genre lyrico-narratif en vogue au XIII ${ }^{\mathrm{e}}$ siècle:

Il li a les cuissees overtes,

Et quant les soies i a mises,

Les flors del pucelage a prises ${ }^{18}$.

Il lui a ouvert les cuisses

Et y a glissé les siennes,

Cueillant la fleur de sa virginité.

La soumission de l'inconnue à la violence du désir masculin n'est toutefois qu'apparente. Mélior précise après-coup qu'elle ne s'est nullement laissée aller à un acte de "folie» (v. 1325) et qu'elle a droit au respect et à l'amour de Partenopeu. La fée révèle qu'elle a tout orchestré par son «engien» (v. 1381), envoyant le sanglier au héros dont elle est amoureuse, puis l'attirant dans son palais et dans son lit. De tels pouvoirs inquiètent à juste titre son amant, mais la profession de foi de Mélior (v. 1535 sqq.) lève, du moins momentanément, son angoisse, car elle fait d'elle - comme plus tard de Mélusine - une bonne chrétienne, avec qui le héros peut entrer en relation. Les deux fées, qui offrent richesse et amour à l'homme, ont des attributs habituellement dévolus à Fortune; elles corrigent la réalité en donnant un sens à la vie du héros. La fertilité

I5. Cf. Ch. Ferlampin-Acher, Merveilles et topique merveilleuse dans les romans médiévaux, p. 13-17.

I6. La dame de l'Autre Monde est, comme le relève M. Dessaint, «une femme libre que ne rebute nullement la sexualité» (La Femme médiatrice dans les grandes auvres romanesques $d u X I I^{e}$ siècle, p. 62).

17. Le rapprochement a été proposé par D. Hüe, «Faire d'armes, parler d'amour», p. 112-113. Une scène presque identique est citée et commentée par K. Gravdal, "Camoufling Rape», p. 366.

18. Partenopeu de Blois, v. 1298-1300. 
de la fée, écrit Friedrich Wolfzettel ${ }^{19}$, est la fertilité du folklore et de la troisième fonction dumézilienne, liée à la terre, la richesse et la sexualité. Mais on perçoit aussi chez Mélior et chez Mélusine les reflets des fata, des Parques antiques: comme dans les récits et les rites célébrant la victoire d'un saint sur un dragon, étudiés par Jacques Le Goff ${ }^{20}$, l'opposition entre origine savante (écrite) et substrat folklorique (oral) est difficile à cerner, car les deux types de discours se répondent, se contaminent, voire s'amalgament sous la plume des clercs.

Bien plus tard, alors que Partenopeu vient de transgresser l'interdit, il apprend de la bouche de Mélior qu'elle est la fille de l'empereur de Constantinople (v. 4559), lieu de la merveille où prennent forme les rêves de richesse et de puissance des Occidentaux ${ }^{21}$. La princesse allie séduction, pouvoir politique et savoir universel ${ }^{22}$, car elle maîtrise les sept arts libéraux, "tote mecine» (v. 4583) et elle a une connaissance approfondie des Ecritures saintes. Comme Mahomet (v. 4607) enfin - le rapprochement est inquiétant! ${ }^{23}$-, Mélior recourt à la "nigromance» ${ }^{24}$. Le long discours de l'héroïne se fait ainsi "l'écho d'une polysémie» ${ }^{25}$, à travers laquelle s'exprime sa profonde ambiguïté; elle s'apparente aux fées qui, dans certains lais et romans, sont perçues comme des «figures surnaturelles chrétiennes, mais non orthodoxes et pourvues de pouvoirs "magiques", à connotation diabolique, tout en étant de parfaites aristocrates" ${ }^{26}$. Les "encantemens» (v. 4598) de Mélior empêchent que Partenopeu n'aperçoive les habitants de Chief d'Oirre qui eux-mêmes ne se rendent pas compte de sa présence. Cette toute-puissance de la fée se

I9. F. Wolfzettel, Le Conte en palimpseste, p. 26: «Die Fruchtbarkeit der Fee ist auch die Fruchtbarkeit der Folklore und der dritten Funktion.»

20. J. Le Goff, "Culture ecclésiastique et culture folklorique au Moyen Age", p. 264-267.

2I. P. Zumthor, La Mesure du monde, p. 113-114.

22. C'est là un trait récurrent des fées: cf. M. Dessaint, La Femme médiatrice dans les grandes auvres romanesques du XIIe siècle, p. 37-43.

23. Sur Mahomet, "canaille doublée d'un hérésiarque», auteur de faux miracles grâce à la magie noire, voir J. Tolan, Les Sarrasins, p. 200-202.

24. F. Gingras (Erotisme et merveilles dans le récit français des XII et XIII siècles, p. 137) voit dans le recours à la magie noire une manifestation d'une féminité inquiétante.

25. Ch. Ferlampin-Acher, Merveilles et topique merveilleuse dans les romans médiévaux, p. 403.

26. A. Guerreau-Jalabert, «Des Fées et des diables», p. 114. 
limite toutefois à la capacité d'assurer le secret de leur amour: tout son «engin» se révèle inefficace, quand il s'agit de contrecarrer les projets de la mère de Partenopeu. Les pouvoirs de Mélior prennent fin, à peine son ami a-t-il transgressé l'interdit et que, le lendemain matin, les amants sont surpris au lit par l'entourage de la princesse:

Tot ai perdu par vos assaus.

Ains que li jors puisse paroir

Savrés molt bien que jo di voir,

Car ne vos puis mais plus celer

Fors seulement dusque al jor cler $^{27}$.

A cause de vous, j'ai tout perdu.

Avant le lever du jour,

Vous saurez bien si je dis vrai,

Car je ne pourrai plus vous cacher

Que jusqu'à l'aube.

La critique a été frappée par ce brusque changement, suite auquel la fée se transforme en une princesse impuissante dans un monde dominé par les hommes. Dans la seconde partie du récit, Mélior dépend en tout de la décision de ses barons et assiste en spectatrice au tournoi qui permettra à Partenopeu de la reconquérir. Alors qu'elle était au début une amante passionnée et entreprenante, qui avait su se créer un espace privé de liberté, elle n'ose plus - après le scandale - exprimer ses sentiments en public, dire sa préférence pour le chevalier qu'elle aime. On a vu dans cette rupture une volonté de récupérer la fée ${ }^{28}$, de l'intégrer à la société en permettant au chevalier de l'obtenir par les armes, puis de lui assigner sa place par le mariage. Dans une société où les décisions incombent aux hommes, telle est la thèse dominante ${ }^{29}$, la fée doit être privée d'un «engin» qui ne laisse pas d'inquiéter, puisqu'il implique, comme le veut son étymologie (ingenium), à la fois la ruse et l'intelligence. Mélior doit

27. Partenopeu de Blois, v. 4656-4660.

28. Voir notamment C. P. Donagher, "Socializing the Sorceress».

29. Au XII ${ }^{\mathrm{e}}$ Congrès International de la Société Courtoise (Lausanne et Genève, 29 juillet - 4 août 2007), A. Reynders a discuté dans une communication (Les Femmes à la cour dans le Roman de Partenopeu de Blois et ses réécritures. Une analyse de la (re) construction du rôle social de Mélior) les positions de C. P. Donagher (voir note 28), de M. T. Bruckner (Shaping Romance, p. 109-155) et de G. Mieszkowski ("Urake and the Gender Roles of Partenope of Blois»). 
être tirée de la nuit où triomphe son savoir et dont le voile confère au corps féminin son pouvoir mystérieux de séduction ${ }^{30}$. L'ordre féodal ne saurait tolérer un monde parallèle, dans lequel peut se vivre un amour hors la loi.

Les oppositions privé/public et masculin/féminin n'expliquent pourtant pas tout. Dans la première partie du récit, le narrateur ne cesse de rappeler la jeunesse de Partenopeu. Le neveu du roi de France n'a que treize ans (v. 543) et la peur du héros face aux merveilles de Chief d'Oirre est celle d'un "enfes " ${ }^{31}$. La nuit d'amour unit un "damoisel» (v. 1017) à une «damoisele» (v. 1129), de sorte que l'espace secret créé par la fée s'identifie à l'intimité de la chambre, lieu réservé à la découverte de la sexualité par deux adolescents. Le début du roman interpelle par cette «conjoncture existentielle» qui, aux yeux de Bruno Bettelheim ${ }^{32}$, confère aux contes de fée leur force de fascination; il a l'attrait de ces "situations qu'on éprouve», selon Yves Bonnefoy, "fondamentales, aussi énigmatiques demeurent-elles» ${ }^{33}$, quand on lit les romans bretons.

Le caractère fusionnel et exclusif des premières amours ne dure guère dans les récits du Moyen Age. Comme dans les romans dits «idylliques» ${ }^{34}$, la séparation des jeunes amants provoque une réorientation de l'aventure et conduit à une redéfinition des valeurs amoureuses. Que ce soit Partenopeu de Blois ou, plus tard, Galeran de Bretagne dans l'œuvre homonyme de Jean Renart ${ }^{35}$, le héros chassé du paradis des amours juvéniles découvre l'importance de l'attente, il connaît la souffrance du désir inassouvi. L'exploit chevaleresque s'offre alors comme voie d'issue, car le tournoi et la guerre permettent à Partenopeu d'obtenir la reconnaissance sociale, sans laquelle il ne pourrait pas briguer la main de Mélior. L'âge adulte demande un rééquilibrage entre les aspirations individuelles et les exigences de la société, il impose à l'homme et à la femme de canaliser leurs désirs. La disparition de la merveille va ainsi de pair avec la fin de l'adolescence: Partenopeu de Blois s'ouvre sur le récit d'une initiation

30. Voir à ce sujet F. Gingras, Erotisme et merveilles dans le récit français des XII et XIII' siècles, p. 392-394.

3I. Partenopeu de Blois, v. 807 : cf. v. 873, 904, 1091, 1116, 1140 («tousel»), 1155, etc.

32. B. Bettelheim, Psychanalyse des contes de fées, p. 18-25.

33. Y. Bonnefoy, "L'Attrait des romans bretons", p. 128.

34. Le terme est emprunté à M. Lot-Borodine, Le Roman idyllique au Moyen Age.

35. Cf. A. Sobeczyk, L'Erotisme des adolescents dans la littérature française du Moyen Age, p. 136-146. 
sous l'égide de la fée, puis raconte le passage difficile à la maturité, de la découverte de la sexualité aux compromis que la vie impose à l'adulte. A la féerie, au rêve de l'idylle intime, fait suite une utopie sociale ${ }^{36}$ traversée par le désir de recréer la merveille des débuts au sein du mariage. Seulement, si le retour au statu quo ante est possible dans le roman «idyllique", dont le but premier est de restaurer le paradis perdu ${ }^{37}$, le couple impérial ne sort pas indemne des aventures dans Partenopeu de Blois. A la fin du récit, ce sont deux adultes qui se retrouvent et la société les a entretemps contraints à redéfinir leur rôle respectif. Leur bonheur, fragile et humain, est désormais soumis aux dures lois du temps et de l'histoire.

Une telle lecture, basée sur des indices textuels convergents, ne correspond pourtant qu'en partie à l'horizon d'attente créé par le prologue de l'œuvre. C'est en clé littéraire que le narrateur nous invite à aborder le roman : la triple ouverture printanière, inspirée de la "reverdie» lyrique, ne se limite pas, comme le pense Annie Combes ${ }^{38}$, à justifier la rédaction du Partenopeu. Elle n'a pas seulement une fonction "factitive", car elle annonce la découverte, par le héros, de la souffrance née du désir inassouvi en la plaçant d'entrée sous le signe de la fin'amor chantée par les troubadours. Au chant des trois oiseaux - l'alouette, le rossignol et le loriot - correspondent trois conceptions différentes de l'amour, à travers lesquelles l'auteur revendique face au lecteur la «littérarité» de son œuvre.

L'alouette, qui salue «l'aube del jor» (v. 22), invite à penser sans cesse à sa «bele et buene amie» (v. 28), comme le fait tout amant courtois, intériorisant l'image de celle qu'il aime. Son chant lasse toutefois, parce qu'elle en fait un trop grand étalage. Pendant la belle saison, le rossignol égrène ses «lais» (v. 31) nuit et jour, mais il se tait, quand il n'est plus temps de chanter. Capable d'attendre l'heure propice à l'amour, il laisse transparaître la crainte des "losengiers", de ces médisants que dénoncent trouvères et troubadours. Quant au loriot, rare dans les «reverdies» ${ }^{39}$, il siffle doucement, car il évoque, dans une rime topique aux échos

36. C'est la conclusion à laquelle arrive aussi, dans son étude sur le roman idyllique, M. Vuagnoux-Uhlig, Le Couple en herbe, p. 425-430.

37. Voir à ce sujet F. Wolfzettel, «Le Paradis retrouvé», p. 62-68.

38. A. Combes, «La Reverdie», p. 141-142.

39. Le loriot apparaît, en compagnie de la grive, du geai et du rossignol, chez Jean Renart (Galeran de Bretagne, v. 1983-1987) et il chante en compagnie des autres oiseaux dans le jardin de la fée chez Renaut de Beaujeu (Le Bel Inconnu, v. 4324-4328). Comme le montre M. Hennard Dutheil de la Rochère dans le présent volume, l'écrivain 
tristaniens, la «douce dolor» d'une «lointaine amor " ${ }^{40}$. De l'indiscrétion de l'alouette, on passe à la mise en garde du rossignol pour finir par le chant lié aux souffrances causées par la séparation; c'est la courbe que suivront les amours de Mélior et de Partenopeu. La jouissance sans limites prend fin quand le jeune homme, transgressant l'interdit, abolit la limite entre la sphère privée et la sphère publique. Séparé de son amie, tout à sa douleur, il se transforme en amant martyr et fuit la société pour souffrir en silence. Le loriot, qui «soef flahute et a seri» (v. 51), devient l'emblème d'une perfection aussi bien amoureuse que poétique ${ }^{41}$, celle de la vraie courtoisie.

A la seconde partie du roman, placée sous le signe de la fin'amor, s'opposent les rencontres nocturnes des jeunes amants qui suivent un gradus amoris inversé ${ }^{42}$, puisqu'ils commencent par l'acte et finissent par la vue. Emblématiquement, ces rencontres s'interrompent à l'aube, comme dans les pièces lyriques ${ }^{43}$ du même nom: dans ces poésies, le chant des oiseaux annonce la fin de l'espace fugitif de liberté que la nuit offre au couple, car la société impose à nouveau sa loi avec le lever du soleil. La rupture marquée par la disparition de la fée dans Partenopeu de Blois apparaît ainsi comme une rupture registrale: à la chanson de femme, dont l'aube est une des variétés fondamentales, se substituent la logique du grand chant courtois et le point de vue masculin, expression de la doxa féodale. La triple ouverture printanière invite à lire le roman comme un art d'aimer où la passion se déploie sous ses différentes facettes; elle nous propose de mesurer chaque manifestation de l'amour à l'aune des trois postures d'amant évoquées dans le prologue.

Le couple de Mélior et de Partenopeu est entouré d'autres couples qui se font et se défont au fil du récit. Double malheureux du héros, le sultan Margaris ne peut qu'exhaler la douleur d'un amour lointain ${ }^{44}$

contemporain Angela Carter détourne le bestiaire courtois dans sa réécriture de La Belle au bois dormant, qui ouvre elle aussi sur un réel désenchanté.

40. Partenopeu de Blois, v. 53-54.

4I. Voir à ce sujet les remarques d'A.-M. Bégou-Balle, "L'Oiseau chanteur", p. 64-66.

42. Cf. J. Ch. Bateman, «Problems of Recognition», p. 168-169.

43. Pour une définition de ce genre lyrico-narratif, voir P. Bec, La Lyrique française au Moyen Age, vol. I, p. 90-107.

44. Partenopeu de Blois, v. 13669-13976: le salut d'amour ne se trouve que dans le manuscrit $\mathrm{T}$, lequel date du XIV ${ }^{\mathrm{e}}$ siècle. 
dans la lettre qu'il adresse à l'inflexible Mélior. Celle-ci a d'ailleurs aussi ses rivales: à sa suivante Persewis, qui ne peut avouer son amour pour Partenopeu, s'oppose la nièce du roi de France qui tente de le séduire. Seulement, la princesse échoue là où la fée réussit, bien que, comme celle-ci, elle fasse appel aux plaisirs des sens (la table et la sexualité) pour retenir le jeune homme auprès d'elle. Les deux programmes de séduction ne sont pourtant identiques qu'en apparence. La fée agit au nom de son seul amour, s'offrant à l'homme qu'elle a choisi; la princesse agit sur ordre de son oncle et de la mère du héros. Le plaisir des sens n'est pas ici le fruit de la générosité, il n'est pas l'expression du don de soi. Il s'agit d'une stratégie mensongère, par laquelle la nièce du roi de France s'apparente aux femmes, rusées et calculatrices, des fabliaux. Partenopeu de Blois se lit comme une suite de séquences consacrées aux manifestations de l'amour et de l'érotisme: à un extrême, on trouve le traître qui détruit la liaison entre Ansel et la fille de l'empereur de Rome ${ }^{45}$, tentant d'obtenir pour lui-même les faveurs de la jeune fille; à l'autre extrême, on suit l'ascèse imposée au fin amant, par laquelle la passion illicite des jeunes gens est sublimée et devient socialement acceptable. La valorisation de l'amour courtois est confirmée par le narrateur qui, à l'instar du loriot, adopte la posture lyrique du soupirant. Il espère en vain les faveurs d'une dame que sa perfection rend lointaine et inaccessible:

S'el eüst une teche en soi

Si con j’ain li qu'ele aimast moi,

Donc n'i eüst il que refaire,

Mais ce me fait de morne cheire

Que ne la puis metre en s'entente

Qu'el me vuelle faire consent $[\mathrm{t}] \mathrm{e}^{46}$.

Si seulement elle était capable

De maimer comme moi je l'aime,

Ce serait parfait,

Mais cela m'attriste

Que je ne puisse pas la convaincre

De me donner satisfaction.

45. Partenopeu de Blois, v. 11239-11682 (manuscrit T).

46. Partenopeu de Blois, v. 9219-9224 (manuscrit B). 
La «translittération", qui conduit de l'Asinus aureus à Partenopeu de Blois, montre jusqu'où va le processus d'appropriation d'un motif ${ }^{47}$ que nous percevons comme archaïque ou folklorique. L'auteur lui confère un statut littéraire en l'insérant dans un système inédit de relations, de manière à adapter au goût de son public aristocratique le récit auquel l'écriture, puis la lecture actualisante ${ }^{48}$, offrent une seconde vie. Le roman médiéval se lit et s'éclaire à la lumière des textes contemporains sur l'amour. Son auteur anonyme décrit une échelle amoureuse, sur laquelle l'érotisme adolescent sert de prélude à l'ascèse courtoise qui, elle-même, aboutit au mariage. A chaque étape correspond un registre littéraire différent: on a reconnu dans la première partie la structure morganienne ${ }^{49}$ propre à bien des lais féeriques ${ }^{50}$; la seconde suit la logique de la quête chevaleresque, telle qu'on la connaît du roman arthurien, tandis qu'après le mariage domine la veine épique (le combat contre les Sarrasins), dans laquelle les amours de Mélior et de Partenopeu n'ont plus guère de place.

Le rapprochement suggéré ici entre la première partie de Partenopeu de Blois et les lais pourrait inciter à croire que, du début à la fin, ces derniers obéissent nécessairement à la seule logique féerique. Or, ceci n'est pas toujours le cas, comme le rappelle l'exemple du lai de Guigemar de Marie de France, dont la structure est étonnamment proche de celle qui caractérise notre roman. La poétesse commence par créer une atmosphère merveilleuse pour, ensuite, l'évacuer et décevoir notre attente en ne respectant pas le schéma morganien qu'elle utilise en ouverture, avant de rationaliser la fée dans la seconde partie du lai. Un jour qu'il s'est enfoncé dans la forêt pour chasser, Guigemar découvre une biche blanche - couleur de la féerie dans la tradition celtique - dont le front est orné de bois de cerf. Il tire, mais sa flèche, ricochant sur l'animal androgyne, le blesse à la cuisse ${ }^{51}$. La biche prophétise alors qu'il sera guéri seulement par une femme qui souffrira d'amour pour lui. Errant comme

47. Au sens (parcours narratif stéréotypé) que lui donne J.-J. Vincensini, Motifs et thèmes $d u$ récit médiéval, p. 58-78.

48. Y. Citton, Lire, interpréter, actualiser, p. 273.

49. Sur les récits morganiens, cf. L. Harf-Lancner, Les Fées au Moyen Age, p. 203-214.

50. Déjà au Moyen Age, Denis Piramus a rapproché Partenopeu et les Lais de Marie de France: cf. D. Rieger, "Evasion et conscience des problèmes dans les Lais de Marie de France», p. 67-69.

51. Sur les connotations sexuelles de la blessure, cf. M. Mikhaïlova, Le Présent de Marie, p. 67-68. 
Partenopeu dans la forêt, Guigemar arrive à un bras de mer où une nef mystérieuse, sans personne à bord, l'attend. Il y monte et est malgré lui emmené jusqu'à une île où il aborde "ainz la vespree» 52 .

La démultiplication d'éléments merveilleux dans le récit de Marie de France annonce, aux yeux du lecteur, la rencontre avec la fée. Mais Guigemar sera soigné par une "malmariée», par l'épouse malheureuse d'un vieux jaloux, qui prend peur en voyant arriver la nef sans équipage. Au contraire de Mélior, elle ignore tout de l'arrivée de l'inconnu et ne dispose d'aucun savoir particulier. La guérison de Guigemar ne doit rien à des pouvoirs magiques, mais tout à l'amour. Pendant une année et demie, la dame réussit à cacher celui à qui elle a donné son cœur. Mais après ce temps de bonheur, le mari outragé chasse Guigemar et la dame profite de la nef, qui réapparaît au moment opportun, pour partir à sa recherche. Elle est retenue prisonnière par un seigneur, jusqu'à ce que Guigemar ne la libère et l'épouse après l'avoir reconnue lors d'une fête.

Dans le lai de Guigemar, le merveilleux, limité à l'ouverture du récit, puis évacué, tourne en quelque sorte à vide. Marie de France ne nous transporte pas dans un Ailleurs féerique et utilise les motifs hérités de la tradition celtique comme autant de métaphores pour exprimer la naissance de l'amour ${ }^{53}$. La double blessure, celle de la biche et du héros, annonce la blessure d'amour dont seront victimes Guigemar, jusqu'alors réfractaire à tout sentiment, et la dame, enfermée dans un monde où la passion n'a pas pu éclore. La nef d'une richesse inouïe, avec ce lit où le héros «s'endort» (v. 203), se transforme sous la plume de Marie de France en l'image du désir qui pousse l'homme vers la femme et, plus tard, la femme vers l'homme, prélude à la construction d'un couple où homme et femme sont égaux.

Comme Partenopeu, Guigemar part, sans le savoir, à la découverte de sa sexualité. Il est significatif que la chapelle du château où habite la dame soit ornée de fresques, sur lesquelles on voit Vénus jeter au feu «le livre Ovide» (v. 239), celui - les Remedia Amoris! - où l'auteur enseigne, précise le texte, à combattre l'amour. Le lai aussi bien que le roman proposent une réflexion sur l'amour à travers une figurativité littéraire; l'un

52. Marie de France, Guigemar, dans Lais de Marie de France, v. 205.

53. Cf. F. Dubost, «Les Motifs merveilleux dans les Lais de Marie de France», p. 73;

R. T. Pickens, "Marie de France and the Body Poetic», p. 137. 
et l'autre se prêtent à une lecture en clé psychologique ${ }^{54}$. Ils retracent l'éveil des sens chez un adolescent, puis la construction d'un amour partagé qui, après un temps d'épreuves, est finalement admis par la société. Le merveilleux, chez Marie de France, sert d'embrayeur à une aventure tout humaine; il lui permet aussi, en recourant à des représentations emblématiques et familières d'atteindre cette brièveté à la fois allusive et suggestive, si caractéristique de son écriture.

Partenopeu de Blois, qui pratique volontiers l'amplificatio, ne partage pas l'idéal de brièveté que suit Marie de France. Il la rejoint néanmoins sur un point essentiel en utilisant la merveille comme un embrayeur qui, une fois le récit lancé, peut être évacué. Contrairement à ce qui se passe dans Guigemar, où la dame n'est pas dotée d'attributs féeriques, Mélior participe à la mise en place du merveilleux avant qu'elle ne perde ses pouvoirs. Chez Marie de France, la dame, mariée, malheureuse et faible, est d'emblée saisie dans son humanité souffrante. De son malheur même naît le désir (mimétique) qui la pousse à suivre l'exemple de Vénus et à braver les interdits sociaux pour se forger une réalité conforme à celle que lui fait miroiter la littérature. Mélior, jeune fille encore vierge, incarne le mystère de la féminité. La fée vit la même métamorphose que son amant, découvrant sa sexualité en même temps que lui la sienne. Les deux récits se rejoignent dans la mesure où le merveilleux y sert d'allégorie à un vécu humain, car Partenopeu de Blois a beau consacrer quelque 600 vers à l'aventure merveilleuse, l'auteur ne s'intéresse guère au surnaturel pour lui-même, qu'il réduit à un prélude au roman. Au-delà de la fascination que le passage exerce sur le lecteur moderne, peu sensible aux descriptions des combats qui suivent, l'effet de dépaysement reste passager. L'Altérité est d'ailleurs moins radicale qu'on ne serait tenté de le croire: malgré son éclat extraordinaire aux couleurs orientales, qui la distingue du cadre français, familier au public, dans lequel Partenopeu a grandi, Chief d'Oirre ressemble étonnamment à une ville médiévale. Elle est à la fois le reflet idéalisé de la réalité et une projection subjective du héros, à travers les yeux duquel nous la découvrons. Confinée dans un espace nocturne et irréel, voire onirique, la fée n'est pas une réalité ontologique dans Partenopeu de Blois: elle est une fonction, "una bella menzogna " 55

54. Comme le fait E. Sienaert, Les Lais de Marie de France, p. 51-67.

55. Ce sont les termes utilisés par Dante pour définir les fables des poètes (Convivio, II, 1). 
dont se sert le poète pour éveiller la curiosité du public et l'amener par la suite à réfléchir - sous le voile de la fiction - au difficile équilibre entre les valeurs amoureuses, expression des aspirations individuelles, et les valeurs sociales, sur lesquelles repose la stabilité du royaume.

Tout autre est le statut de la fée dans Le Roman de Mélusine que Jean d'Arras rédige en 1393 pour le duc Jean de Berry. Nous y trouvons pourtant la même structure bipartite d'un "conte de fée qui s'ouvre sur le réel ${ }^{56}$ que dans Partenopeu de Blois. Aux amours de Raymondin et de Mélusine fait suite la conquête de nouvelles terres et d'une épouse par leurs fils qui étendent ainsi la domination des Lusignan jusqu'à Chypre et à l'Arménie. Avec les combats contre les Sarrasins, le registre épique domine dans la seconde partie du récit, où les armes imposent leur loi comme elles le font déjà dans le roman du XIIe siècle. Mais, contrairement à Mélior ${ }^{57}$, Mélusine ne peut pas être définitivement intégrée au monde des hommes. Après la disparition de la fée, qui s'envole sous forme de dragon, manifestant publiquement sa nature merveilleuse et inquiétante, commence le déclin des Lusignan. Le temps de l'histoire s'oppose ici au temps des origines, quand la fée providentielle garantissait la richesse et le pouvoir de la famille. A la courbe euphorique que dessine Partenopeu de Blois, où la reconquête du bonheur est possible, s'oppose la courbe dysphorique du roman de Mélusine, dans lequel on reconnaîtra, avec Friedrich Wolfzettel, la structure du "Verlustmärchen » ${ }^{58}$ : le récit de Jean d'Arras véhicule la nostalgie d'un paradis perdu aux connotations mythiques, le poids aussi de la faute originelle qui a causé sa disparition. Il n'y a pourtant pas, chez Jean d'Arras, «de solution de continuité entre le monde empirique et le monde du folklore» 59 . Dans le prologue, il se réclame de l'autorité de Gervais de Tilbury ${ }^{60}$ qui, dès le début du

56. La formule est de F. Wolfzettel (“"Songe” et/ou “histoire” ”, p. 390), qui l'applique à Coudrette dont nous ne parlerons pas ici.

57. Signalons que «Mélior» est à la fois le nom de la fée de Partenopeu et de l'une des sœurs de Mélusine. L'une et l'autre habitent un château mystérieux en Orient. Les ressemblances, il est vrai, s'arrêtent là.

58. F. Wolfzettel, "Songe" et/ou "histoire" ", p. 391.

59. F. Wolfzettel, «La "Découverte” du folklore et du merveilleux folklorique au Moyen Age tardif», p. 628.

6o. Sur les récits "pré-mélusiniens" rapportés par des clercs, porte-paroles du point de vue ecclésiastique, voir A. Guerreau-Jalabert, «Des Fées et des diables», p. 115-124; 
XIII ${ }^{\mathrm{e}}$ siècle, considérait dans les Otia imperialia que les mirabilia font partie de la réalité et doivent par conséquent être répertoriés. Son témoignage rejoint, sous la plume de Jean d'Arras, les dires des «anciens" qui affirment avoir rencontré des fées et des lutins dans la campagne poitevine. La concordance entre l'écrit et l'oral, entre l'érudition du clerc et l'expérience du peuple, incite l'auteur de Mélusine à prendre au sérieux les récits des témoins oculaires. Nous sommes loin des superstitiones aniles dont se moquait Cicéron ${ }^{61}$, quand il dénonçait l'inanité des fables mythologiques:

Nous avons ouy raconter a noz anciens que en plusieurs parties sont apparues a pluseurs tresfamillierement choses lesquelles aucuns appelloient luitons, aucuns autres les faes, aucuns autres les bonnes dames qui vont de nuit ${ }^{62}$.

Folkloriste avant la lettre ${ }^{63}$, Jean d'Arras fait du travail sur le terrain en recueillant des témoignages oraux. Il affirme avoir vu lui-même des «choses que pluseurs ne pourroient croire sans le veoir" ${ }^{64}$ et est convaincu qu'un voyageur attentif, parcourant provinces et royaumes, ne manquera pas de se retrouver face à des phénomènes qui dépassent son entendement, mais qui n'en sont pas moins vrais. Comme le dit saint Paul, dont l'autorité est invoquée pour justifier la croyance en l'existence des fées, les chemins du Seigneur sont impénétrables. En choisissant la prose $^{65}$ - et non pas le vers, comme le fera Coudrette - Jean d'Arras suit

sur l'attitude scientifique de Gervais face à la merveille, voir F. Dubost, Aspects fantastiques de la littérature narrative médiévale, p. 43-45.

6I. Cicéron, De natura deorum, 2.70: "des superstitions de vieilles femmes".

62. Jean d'Arras, Mélusine ou La Noble Histoire de Lusignan, p. 116: «Nous avons donc entendu nos anciens rapporter qu'en diverses régions sont apparus en toute familiarité à diverses personnes ces êtres de la nuit que certains appellent des lutins, d'autres des fées, d'autres encore les bonnes dames" (p. 117).

63. Cf. F. Wolfzettel, Le Conte en palimpseste, p. 151-164 (surtout p. 153). Malgré des sous-titres prometteurs ("Qui croit aux fées?», p. 115; "Un conte... de fée?», p. 197), M. White-Le Goff, Envoûtante Mélusine, n'apporte rien à la réflexion: l'ouvrage, qui vise un large public, s'appuie sur une bibliographie plus que sélective et ignore les propositions de Friedrich Wolfzettel.

64. Jean d'Arras, Mélusine ou La Noble Histoire de Lusignan, p. 118.

65. Sur l'opposition vers/prose à la fin du Moyen Age, on consultera la thèse de L. Raffalli-Grenat, Ecrire des fictions en vers aux XIV et XVe siècles, un problème esthétique et culturel, partie II ("Vers et prose: comparaison des romans de Mélusine et de Richard sans peur»). 
le modèle historiographique et souligne ainsi le caractère véridique d'une aventure qui, emblématiquement, s'ouvre sur: "Il est verité qu'il ot jadis un roy en Albanie ...» ${ }^{66}$.

L'épilogue fait écho au prologue en mobilisant encore une fois saint Paul et Gervais de Tilbury. Jean d'Arras y apporte une preuve supplémentaire qu'il raconte une "ystoire veritable" ${ }^{67}$ (p. 810) : il ancre son récit dans la réalité et dans une région - comme l'est habituellement le folklore - lorsqu'il évoque l'aventure vécue par Creswell, capitaine anglais qui occupait la forteresse de Lusignan en 1373-1374 au nom du roi Edouard III :

Il vit, ce disoit il, apparoir presentement et visiblement devant son lit une serpente grande et grosse merveilleusement, et estoit la queue longue de .vii. a .viii. piéz, burlee d'azur et d'argent ${ }^{68}$.

La serpente, avec sa queue aux couleurs héraldiques des Lusignan, est identifiée par les témoins comme la dame qui a jadis fait construire le château. Mélusine vient signifier à l'occupant qu'il est temps de quitter les lieux... pour laisser la place au duc de Berry, comte de Poitou. Ce prince est instauré par la fée en héritier légitime ${ }^{69}$ d'une famille dont le dernier descendant direct, Léon de Lusignan, roi d'Arménie, meurt en exil à Paris en 1393, au moment même où Jean d'Arras termine son roman et que son mécène négocie avec les Anglais pour garder son apanage.

Le roman de Mélusine offre un exemple exceptionnel de la revalorisation du folklore, perçu dans sa différence (son oralité, son origine populaire) en cet automne du Moyen Age et, corollairement, de sa récupération à des fins politiques. Jamais l'auteur anonyme de Partenopeu de Blois ni Marie de France n’auraient accordé un tel crédit à des témoins oculaires pour affirmer sans ambages l'historicité des fées. Ils restent proches de Wace, leur contemporain, qui déclare dans Le Roman de Rou n'avoir découvert aucune merveille à la fontaine de Barenton, alors que les Bretons affirment que,

66. Jean d'Arras, Mélusine ou La Noble Histoire de Lusignan, p. 120.

67. Ibid., p. 810.

68. Ibid., p. 810: «Il vit, dit-il, apparaître distinctement à sa vue, juste devant son lit, une serpente fabuleusement grande et grosse; sa queue, burelée d'azur et d'argent, faisant bien sept à huit pieds de long» (p. 811).

69. Sur les implications politiques du récit, voir D. Delogu, «Jean d’Arras makes History", p. 23-26. 
jadis, on y "seut les fees veeir " ${ }^{70}$. Pour le clerc normand, ces récits ne sont que «folie» (v. 6398) et les fées des êtres nés de l'imagination des hommes. De même, chez Marie de France et dans Partenopeu de Blois, le merveilleux donne au récit les couleurs de la fable: leur manière de s'en servir pour exprimer un drame humain sous le voile de la fiction annonce, mutatis mutandis, l'attitude de Madame d'Aulnoy qui, en 1697, fait précéder ses Contes d'une lettre dédicatoire, selon laquelle l'exemple des "grandes princesses» aurait "donné lieu d'imaginer le royaume de féerie » ${ }^{71}$.

Chez Jean d'Arras, au contraire, le merveilleux fait partie de la réalité et il vient se mêler de manière presque imperceptible au quotidien des hommes. Alors que le grand sanglier, qui égare Partenopeu dans la forêt, échappe au jeune homme et lui ouvre les portes de l'Ailleurs, Raymondin, lui, tue le sanglier en même temps que son oncle dans Le Roman de Mélusine. Mort, l'animal ne saurait assumer la fonction de guide faé vers une quelconque nef merveilleuse qui emmènerait le héros dans l'au-delà. L'errance de Raymondin, désemparé après son meurtre, introduit - malgré un scénario inspiré de la tradition celtique - le surnaturel par petites touches, de sorte que le lecteur ne lui accorde pas d'emblée un statut à part, hésitant s'il doit ou non le rattacher à la réalité. La rencontre de Raymondin et de Mélusine à la fontaine de Soif en pleine nuit peut lui apparaître comme un fruit du hasard, car rien ne l'annonce, si ce n'est l'endroit et l'heure insolites. C'est par ses paroles, puis par ses actes que Mélusine manifeste ses pouvoirs et se transforme en représentante du destin et, parallèlement, en déléguée (partielle) d'un narrateur omniscient. Elle lance l'action, puis tire les fils des aventures, comme l'ont fait, avant elle, Lunete dans Le Chevalier au lion de Chrétien de Troyes, la fée de Lanval $^{72}$ ou la Dame aux blanches mains dans Le Bel Inconnu de Renaut

70. Wace, Le Roman de Rou, v. 6387 («on y voyait jadis les fée»). Pour J. Le Goff («Lévi-Strauss en Brocéliande», p. 613), le passage témoigne "de la désacralisation de la forêt défrichée».

7I. M.-C. d'Aulnoy, Contes de fées, p. 109. Rappelons que L'Oiseau bleu raconte la même histoire que le lai de Yonec de Marie de France. D'autre part, Madame d'Aulnoy évoque volontiers l'histoire de Psyché (cf. p. 91, 125, 235, 648). Sur l'intertexte courtois dans l'œuvre de M.-C. d'Aulnoy, voir l'article de S. Ballestra-Puech dans le présent volume.

72. A. Paupert («Les Femmes et la parole dans les Lais de Marie de France», p. 169177) souligne le caractère performatif de la parole féminine chez Marie de France. La remarque vaut aussi pour les fées dans les récits dont il est question ici et explique peutêtre pourquoi le conte de fées se profile peu à peu comme genre littéraire privilégié des 
de Beaujeu. Mélusine révèle sa nature de fée en arrachant le malheureux Raymondin à son cauchemar: elle l'amène à affronter la réalité et, lui montrant comment retourner à son avantage une situation apparemment désespérée, elle renoue les fils rompus du récit - et de la vie.

La rencontre avec Mélior arrache au contraire Partenopeu à la vie quotidienne et le transpose, comme Psyché, dans un monde aux couleurs du rêve. La toute-puissance initiale de la fée dit combien la magie - pour paraphraser Giorgio Agamben ${ }^{73}$ - est nécessaire au bonheur qui prend ici les apparences de la merveille. Le bonheur n'est pas le fruit du mérite et l'intervention providentielle de la fée est le signe d'une élection et de la chance. La seconde partie de Partenopeu rompt avec la logique merveilleuse en nous faisant croire que le bonheur et l'amour peuvent se mériter, qu'on peut les conquérir par l'épreuve, comme le font aussi la malmariée et Guigemar dans le lai de Marie de France. La question du mérite ne se pose pas dans Mélusine: jamais Raymondin ne se libère de l'emprise de l'être venu d'Ailleurs, jamais il ne devient l'artisan de sa vie. Aux côtés de son épouse, il joue un rôle plus qu'effacé et Mélusine oriente le cours des choses aussi après sa disparition. Fée "maternelle et défricheuse " ${ }^{74}$, puis prophétesse des malheurs à venir, bénéfique ou maléfique, elle reste toujours l'agent du destin. Elle ne perd pas son halo mythique, au contraire de Mélior qui devient femme parmi les femmes. Mélusine est l'histoire des Lusignan, qu'elle incarne, et ses retours au fil des siècles, jusque dans un passé récent, démontrent l'existence de la fée tutélaire. Contrairement à l'auteur de Partenopeu de Blois, Jean d'Arras ne fait pas de la merveille un indice de littérarité et de fiction. A l'en croire, les fées vivent parmi nous et le surnaturel fait partie de l'histoire: l'expérience, l'enseignement de la Bible, ne prouvent-ils pas qu'il y a des mondes parallèles et contigus?

\author{
Jean-Claude MüHLethaleR \\ Université de Lausanne
}

\footnotetext{
femmes écrivains, de Marie de France, unique représentante de l'époque médiévale, aux auteurs contemporains. Voir l'ouvrage d'E. W. Harries, Twice Upon a Time, et les articles de D. Haase, M. Hennard Dutheil de la Rochère et M. Monnier dans le présent volume.

73. G. Agamben, «Magie et bonheur», p. 64-65.

74. On aura reconnu le titre de l'article pionnier de J. Le Goff et E. Le Roy Ladurie, "Mélusine maternelle et défricheuse».
} 


\section{BIBLIOGRAPHIE}

\section{Sources}

Apulée, Le Metamorfosi o l'asino d'oro, éd. et trad. par Claudio Annaratone, Milan, BUR, 1977.

Augustin (saint), La Cité de Dieu, éd. et trad. par Pierre de Labriolle et Jacques Perret, Paris, Classiques Garnier, 1941-1946.

Aulnoy, Marie-Catherine le Jumel de Barneville, comtesse d', Contes de fées, éd. par Nadine Jasmin, Paris, Champion Classiques, 2008.

Cicéron, De natura deorum, livre II, éd. par M. Van Den Bruwaene, Bruxelles, Latomus, 1978.

Dante Alighieri, Convivio, II, 1, éd. par Pasquale Papa, Milano, Signorelli, 1968.

Jean D’Arras, Mélusine ou La Noble Histoire de Lusignan, éd. et trad. par Jean-Jacques Vincensini, Paris, Le Livre de Poche (Lettres Gothiques), 2003.

Jean Renart, Galeran de Bretagne, roman du XIII e siècle, éd. par Lucien Foulet, Paris, Champion, 1975.

Marie de France, Lais de Marie de France, éd. par Karl Warnke, trad. par Laurence Harf-Lancner, Paris, Livre de Poche (Lettres Gothiques), 1990.

Partenopeu de Blois, éd. et trad. par Olivier Collet et Pierre-Marie Joris, Paris, Livre de Poche (Lettres Gothiques), 2005.

Renaut de Beaujeu, Le Bel Inconnu, éd. et trad. par Michèle Perret et Isabelle Weill, Paris, Champion Classiques, 2003.

WaCe, Le Roman de Rou, éd. par Anthony J. Holden, Paris, Picard, 1971. 


\section{Travaux}

Agamben, Giorgio, "Magie et bonheur", in Profanations, trad. par Martine Rueff, Paris, Rivages Poche (Petite Bibliothèque), 2005, p. 63-68.

Bateman, J. Chimène, "Problems of Recognition: The Faillible Narrator and the Female Addressee in Partenopeu de Blois", Mediaevalia, 25/2, special issue (2004), p. 163-179.

Bec, Pierre, La Lyrique française au Moyen Age (XII - XIII e siècles), Paris, Picard, 1977.

BÉgou-BAlle, Anne-Marie, "L'Oiseau chanteur: esquisse d'une ornithologie courtoise", Senefiance, 54 (2009: Déduits d'oiseaux au Moyen Age), p. 59-67.

Bettelleim, Bruno, Psychanalyse des contes de fées, Paris, Pocket, 2006.

Bonnefoy, Yves, "L'Attrait des romans bretons", in L'Imaginaire métaphysique, Paris, Seuil, 2006, p. 123-138.

Bruckner, Matilda T., Shaping Romance. Interpretation, Truth and Closure in Twelfth Century French Fiction, Philadelphia, University of Pennsylvania Press, 1993.

Butor, Michel, «La Balance des fées», in Répertoire I, Paris, Editions de Minuit, 1960, p. 61-73.

Citton, Yves, Lire, interpréter, actualiser. Pourquoi les études littéraires?, Paris, Editions Amsterdam, 2007.

Combes, Annie, «La Reverdie: des troubadours aux romanciers arthuriens, les métamorphoses d'un motif ", in L'Espace lyrique méditerranéen au Moyen Age. Nouvelles approches, éd. par Dominique Billy, François Clément, Annie Combes, Toulouse, Presses Universitaires du Mirail, 2006, p. 121-156.

Delogu, Daisy, "Jean d'Arras makes History: Political Legitimacy and the Roman de Mélusine", Dalhousie French Studies, 80 (2007), p. 15-28.

Dessaint, Micheline, La Femme médiatrice dans les grandes auvres romanesques du XII e siècle, Paris, Champion, 2001.

Donagher, Collen P., "Socializing the Sorceress: The Fairy Mistress in Lanval, Bel Inconnu, and Partenopeu de Blois", Essays in Medieval Studies, 4 (1987), p. 69-86. 
Dubost, Francis, Aspects fantastiques de la littérature narrative médiévale (XII ${ }^{e}-X I I I^{e}$ siècles): L'Autre, l'Ailleurs, l'Autrefois, Paris, Champion, 1991.

—, «Les Motifs merveilleux dans les Lais de Marie de France», in Amour et merveille: les Lais de Marie de France, éd. par Jean Dufournet, Paris, Champion (Unichamp), 1995, p. 41-80.

Ferlampin-Acher, Christine, Merveilles et topique merveilleuse dans les romans médiévaux, Paris, Champion, 2003.

Gély, Véronique, L'Invention d'un mythe: Psyché. Allégorie et fiction, du siècle de Platon au temps de La Fontaine, Paris, Champion, 2006.

Gingras, Francis, Erotisme et merveilles dans le récit français des XII et XIII siècles, Paris, Champion, 2002.

Gravdal, Kathryn, "Camoufling Rape: The Rhetoric of Sexual Violence in the Medieval Pastourelle», Romanic Review, 76 (1985), p. 361-373.

Guerreau-Jalabert, Anita, "Des Fées et des diables. Observations sur le sens des récits "mélusiniens” au Moyen Age», in Mélusines continentales et insulaires, éd. par Jeanne-Marie Boivin, Proinsias MacCana, Paris, Champion, 1999, p. 105-137.

Harf-Lancner, Laurence, Les Fées au Moyen Age. Morgane et Mélusine. La naissance des fées, Paris, Champion, 1984.

Harries, Elizabeth W., Twice upon a Time. Women Writers and the History of the Fairy Tale, Princeton, Princeton University Press, 2001.

Heidmann, Ute, Adam, Jean-Michel, Textualité et intertextualité des contes. Perrault, Apulée, La Fontaine, L'Héritier..., Paris, Classiques Garnier, 2010.

HüE, Denis, «Faire d'armes, parler d'amour: les stratégies du récit dans Partenopeu de Blois", Mediaevalia, 25/2, special issue (2004), p. 111-129.

Le Goff, Jacques, "Culture ecclésiastique et culture folklorique au Moyen Age: Saint Marcel de Paris et le dragon", in Un Autre Moyen Age, Paris, Gallimard (Quarto), 1999, p. 229-268.

-, "Lévi-Strauss en Brocéliande", in Un Autre Moyen Age, Paris, Gallimard (Quarto), 1999, p. 581-614.

Le Goff, Jacques, Le Roy Ladurie, Emmanuel, «Mélusine maternelle et défricheuse", Annales E.S.C., 26 (1971), p. 587-622. 
Lot-Borodine, Myrha, Le Roman idyllique au Moyen Age, Genève, Slatkine Reprints, 1972 (1 ${ }^{\text {re }}$ éd., Paris, 1913).

Mieszkowski, Gretchen, "Urake and the Gender Roles of Partenope of Blois", Mediaevalia, 25/2, special issue (2004), p. 181-195.

Mikhaїlova, Milena, Le Présent de Marie, Paris, Diderot, 1996.

Paupert, Anne, "Les Femmes et la parole dans les Lais de Marie de France", in Amour et merveille: les Lais de Marie de France, éd. par Jean Dufournet, Paris, Champion (Unichamp), 1995, p. 169-187.

Pickens, Rubert T., "Marie de France and the Body Poetic", in Gender and Text in the Later Middle Ages, ed. by Jane Chance, Gainesville, University of Florida Press, 1996, p. 135-171.

Poirion, Daniel, "Ecriture et ré-écriture au Moyen Age», in Ecriture poétique et composition romanesque, Orléans, Paradigme, 1994, p. 457-469.

Raffalli-Grenat, Lunorsola, Ecrire des fictions en vers aux XIV et $X V^{e}$ siècles, un problème esthétique et culturel, thèse dirigée par Jean-Jacques Vincensini et soutenue en décembre 2008 à l'Université Pascal Paoli (Corte).

Reynders, Anne, "Le Roman de Partenopeu de Blois est-il l'œuvre d'un précurseur de Chrétien de Troyes?", Le Moyen Age, 111 (2005), p. $479-502$.

Rieger, Dietmar, «Evasion et conscience des problèmes dans les Lais de Marie de France", in Chanter et dire. Etudes sur la littérature du Moyen Age, Paris, Champion, 1997, p. 65-88.

Sienaert, Edgar, Les Lais de Marie de France. Du conte merveilleux à la nouvelle psychologique, Paris, Champion (Essais), 1984.

Sовесzук, Agata, L'Erotisme des adolescents dans la littérature française du Moyen Age, Louvain, Peeters, 2008.

Tolan, John, Les Sarrasins. L'Islam dans l'imagination européenne au Moyen Age, trad. par Pierre-Emmanuel Dauzat, Paris, Aubier, 2003.

Vincensini, Jean-Jacques, Motifs et thèmes du récit médiéval, Paris, Nathan, 2000.

Vuagnoux-Uhlig, Marion, Le Couple en herbe. Galeran de Bretagne et L'Escoufle à la lumière du roman idyllique médiéval, Genève, Droz, 2009.

White-Le Goff, Myriam, Envoûtante Mélusine, Paris, Klincksieck, 2008. 
Wolfzettel, Friedrich, "La "Découverte" du folklore et du merveilleux folklorique au Moyen Age tardif», Le Moyen Français, 51-52-53 (2002-2003: Traduction, dérimation, compilation. La phraséologie), p. 627-640.

-, Le Conte en palimpseste. Studien zur Funktion von Märchen und Mythos im französischen Mittelalter, Wiesbaden, Franz Steiner, 2005.

—, "Songe" et/ou "histoire": Le roman de Mélusine de Coudrette ou le roman conte de fées au carrefour du système générique du Moyen Age tardif", in 550 Jahre deutsche Melusine - Coudrette und Thüring von Ringoltingen / 550 ans de Mélusine allemandeCoudrette et Thüring von Ringoltingen, éd. par André Schnyder, Jean-Claude Mühlethaler, Berne, Peter Lang, 2008, p. 381-394.

—, "Le Paradis retrouvé: pour une typologie du roman idyllique», in $L e$ Récit idyllique. Aux sources du roman contemporain, éd. par JeanJacques Vincensini, Claudio Galderisi, Paris, Classiques Garnier, 2009, p. 59-77.

Zumthor, Paul, La Mesure du monde, Paris, Seuil, 1993. 